\title{
El ascenso turco: entre el éxito económico y la Unión Europea
}

\author{
Andrés Felipe Galindo Farfán* \\ (agalindo72@javerianacali.edu.co)
}

Artículo corto recibido el 03/05/2014 y aprobado el 22/05/2014.

Cómo citar este artículo:

GALINDO FARFÁN, Andrés Felipe (2014). "El ascenso turco: entre el éxito económico y la Unión Europea”. En: Trans-pasando Fronteras, Núm. 5, pp. 85 - 92. Cali, Colombia: Centro de Estudios Interdisciplinarios, Jurídicos, Sociales y Humanistas (CIES), Facultad de Derecho y Ciencias Sociales, Universidad Icesi.

\begin{abstract}
Resumen
Uno de los fenómenos más interesantes de la primera década del siglo XXI ha sido el ascenso vertiginoso de la economía turca, pues ha demostrado un desempeño destacable, especialmente por su crecimiento sostenido. Una serie de estrategias macroeconómicas combinadas con políticas fiscales prudentes y reformas estructurales implementadas desde principios del siglo, han integrado con fuerza a Turquía con un mundo globalizado, han cambiado su estructura económica y la ha convertido en uno de los destinos más importantes de la Inversión Extranjera Directa en la región. No obstante, los desafíos a futuro de esta economía están fuertemente relacionados con el entorno político interno y su eventual adhesión a la Unión Europea, que está determinada por la capacidad del bloque de asimilar y ponderar los costos y beneficios que supone el acceso de una economía emergente de esta naturaleza, con un ingreso per cápita promedio inferior al del bloque europeo.
\end{abstract}

\section{Palabras clave:}

Desempeño, crecimiento económico, reformas, Unión Europea, costos.

\footnotetext{
* $\quad$ Estudiante de Economía con Énfasis en Negocios Internacionales. Pontificia Universidad Javeriana Cali
} 


\section{Introducción: panorama de la economia turca}

En los últimos años el PIB ha mostrado un comportamiento dinámico, lo que ha consolidado a Turquía como una de las grandes economías emergentes a nivel global y con uno de los más elevados potenciales de convertirse en un aportante decisivo para el crecimiento mundial (Babakr, 2013) -especialmente desde el ascenso del Partido actualmente en el Gobierno-; algunos rasgos permiten identificar las características de este dinamismo: i.) un mercado emergente con una gran población, que lo hace un mercado atractivo y, ii.) una estructura económica que ha sido orientada hacia la industria y los servicios, mientras la agricultura y, en general el sector primario, ha ido perdiendo relevancia. Es posible afirmar que los turcos adoptaron las lecciones que dejó la crisis de 2001, que los sorprendió con un sistema financiero débil, con grandes riesgos acumulados y un gobierno fiscalmente vulnerable. La regulación a la que se sometió a los sectores vulnerables de la economía logró reformar el marco institucional del sistema bancario y por esta vía construir la estabilidad financiera.

Los indicadores fundamentales de la economía turca dan cuenta de las principales estrategias que determinaron el desempeño en la última década, los cuales analizarán más adelante. Entre 2001 y 2008 el crecimiento fue bastante favorable, manteniendo un promedio del $5 \%$, con un pico notable en 2005 del 8,4\% y un mínimo del $-4.8 \%$ durante 2009, en medio del impacto de la crisis internacional. No obstante, Turquía pronto recuperó la senda de crecimiento, alcanzando una tasa del 9,2\%, pero con una desaceleración en 2012, donde el crecimiento estuvo cerca del 2,2 \%. Sin embargo, esa rápida capacidad de recuperación luego de las crisis que ha mostrado Turquía parece sugerir que las reformas fiscales, la regulación de sectores claves de la economía y su integración con los mercados internacionales tienen la robustez necesaria para reponerse rápidamente de los ciclos depresivos de la economía. 


\section{Figura 1}

Tasa de crecimiento del PIB. Turquía, 2005-2012

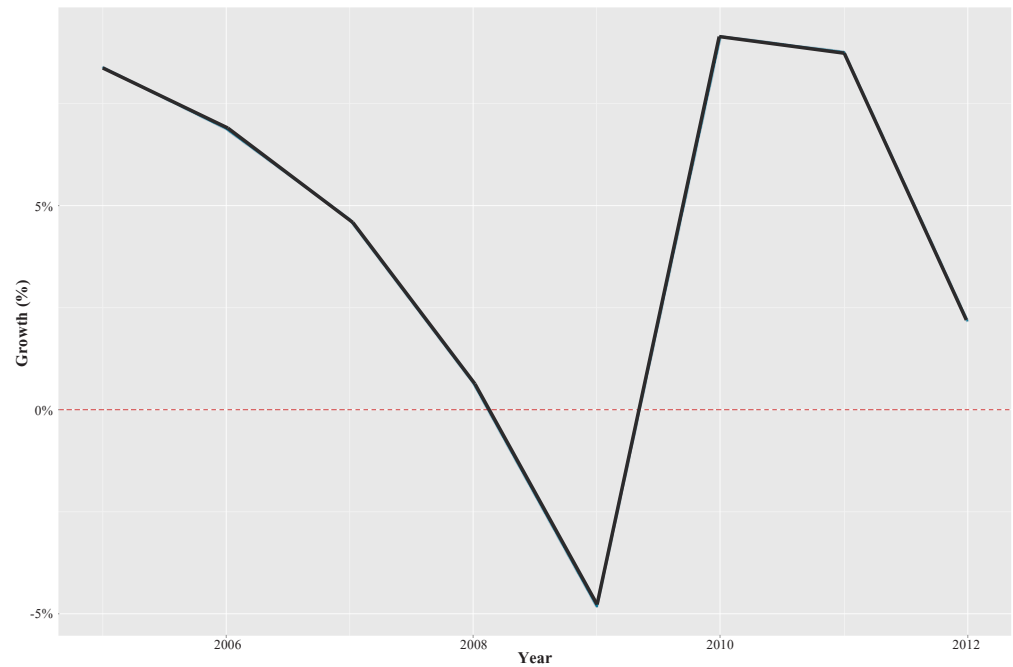

Fuente: $O E C D$.

En gran medida, el buen desempeño macroeconómico de la República de Turquía es la mejor carta de presentación que tiene su Gobierno para impulsar una eventual adhesión a la Unión Europea que, aunque no ha descartado a esta nación como candidata para ingreso al bloque, ha generado resistencias por parte Francia y Alemania. No obstante, la situación presentada a principios de la década pasada es bastante diferente a la que presenta hoy la sociedad turca, que ha visto multiplicar su ingreso per cápita y que padece una tasa de desempleo significativamente inferior que la de países de la UE-28 como España, Grecia y Portugal.

En este trabajo se analizarán tres importantes aspectos que muestran una relación positiva con el desempeño económico mostrado en la última década: en primer lugar, el gasto público y el balance fiscal del Gobierno, donde se revisarán aspectos claves de la política fiscal turca, entre ellas la regla fiscal adoptada a finales de la década pasada.

En segundo lugar, se analizará la dinámica de la Inversión Extranjera Directa (IED) y se intentará relacionar con la dinámica de crecimiento del PIB y los efectos de una tasa de cambio flotante; quizás uno de los aspectos más relevantes de las reformas de 
la pasada década. En tercer lugar, se esbozarán los beneficios de la adhesión de Turquía a la Unión Europea, ponderando los principales aspectos que determinarían una inclusión del mayor economía emergente de la región en el bloque de los 28. Por último, se comentarán los riesgos que pueden impedir que el éxito económico conseguido se estanque, principalmente motivado por la incertidumbre en la política interna.

\section{Consolidación fiscal, reformas y regla fiscal en la primera década del 2000}

A finales del siglo pasado, entre el FMI y el Gobierno se implementaron algunas medidas entre las que estaban una política monetaria orientada a la estabilidad de los precios, que requería con urgencia un sistema de banca central independiente y operacional; la reestructuración del sistema bancario y un esquema de política fiscal conducida hacia la sostenibilidad de las finanzas públicas. Luego de experimentar la crisis de 2001, exacerbada por los ataques terroristas del 11 de septiembre de 2001, en las cuales el Gobierno implementó medidas de austeridad y nuevos impuestos, Turquía terminó con un superávit primario importante, de casi el 4,3 en 2000 y $6 \%$ en 2001. Vale la pena mencionar que en este periodo también se creó el sistema de tasa de cambio flotante (Kaya y Yila, 2011).

Uno de los indicadores más elocuentes de la solidez fiscal mostrada es la capacidad que tuvo posteriormente el Gobierno turco de acceder al crédito en el mercado internacional a costos inferiores. En 2001, los pagos de intereses se situaban en alrededor del $20 \%$ del PIB, frente a menos del $6 \%$ en 2008. Esto, indudablemente, contribuye no sólo a fortalecer la confianza de los mercados internacionales de capital sino a reducir el stock de deuda a niveles aceptables de sostenibilidad.

Del trabajo de Kaya y Yilar (2011), formulado por la OECD, se reconoce la trascendencia de la consolidación fiscal y se señala que Turquía continuó el camino hacia la formulación de nuevas reformas en este sentido. Los objetivos trazados por el Gobierno contenían aspectos esenciales para dar mayor lugar a la rendición de cuentas y la transparencia; abogar por la descentralización presupuestaria, la capacidad de dotar a ciertas agencias gubernamentales de la capacidad de formular sus propios planes estratégicos y la rendición de cuentas; darle mayor lugar al gasto social y; reformar la seguridad social y liberalización de empresas e industrias estatales. En este orden 
Figura 2

Déficit del sector público y Gobierno Central. Turquía, 2000-2008

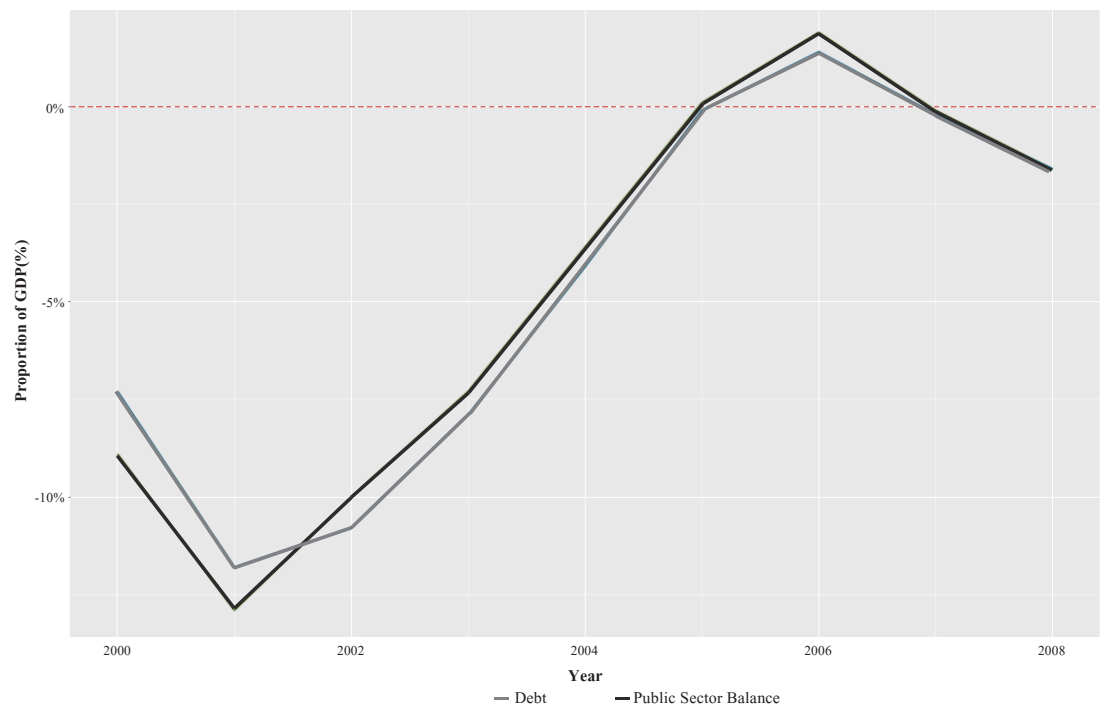

Fuente: $O E C D$.

Figura 3

Evolución de la Inversión Extranjera Directa. Turquía, 20022011

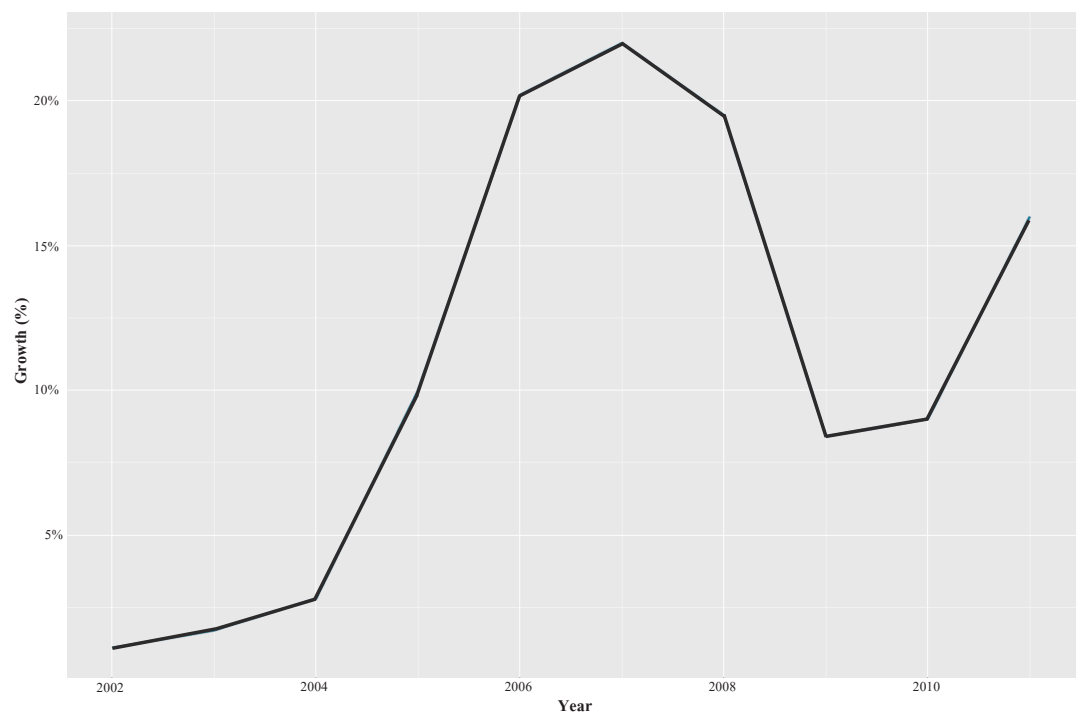

Fuente: $O E C D$. 
de ideas, en el escenario de la política económica emergió el proyecto de regla fiscal, que en gran medida se diseñó ceñida al European System of Accounts, para calcular los déficits de los Gobiernos. En otras palabras, con este arreglo el Gobierno turco demostró la trascendencia de amoldarse a los parámetros del Tratado de Maastricht.

\section{La IED en Turquía: apertura al mundo globalizado}

La teoría económica sugiere la existencia de una relación positiva entre el crecimiento del PIB y la IED. De hecho, algunos trabajos empíricos como los de Koch et al. (2010), han descubierto una relación de causalidad de doble vía entre ambas variables. De hecho, la década de mayor crecimiento de la economía turca coincide con un ascenso vertiginoso, casi exponencial, de los flujos de IED.

Desde 2002 se ha pretendido aprovechar la posición geográfica de Turquía en la confluencia de Asia, Europa y la relativa poca distancia con África. En ese sentido, el Gobierno ha implementado una serie de arreglos legales y estructurales -que no se abordarán en detalle- que han conformado una serie de incentivos para la llegada de inversionistas extranjeros. Un primer impulso fundamental de la IED en Turquía ha sido, posiblemente, en términos de las sucesivas privatizaciones de compañías estatales. Algunas variables adicionales pueden construir mejor un mapa para entender las determinantes de la IED en Turquía: estabilidad política (no ha habido un cambio de partido gobernante en más de una década, aunque ahora hay sombras en torno a la pluralidad política en el país); las relaciones de estado asociado que mantiene Turquía con la UE; bajas tasas de inflación -el Banco Central maneja la política de inflación objetivo-; entre otros aspectos. Sin embargo, la idea es identificar si la IED, motivada por las reformas enunciadas, ha contribuido al éxito turco, específicamente a la expansión de su PIB.

\section{Turquía y la Unión Europea: ¿beneficios mutuos?}

El panorama en los últimos diez años ha cambiado. Los beneficios de la adhesión de Turquía a la UE están balanceados, una década atrás la adhesión para el Gobierno turco era un asunto de prioridad en la agenda, hoy día es un asunto estratégico para ambas partes. Los beneficios económicos de la adhesión para Turquía pueden incluir 
el provecho de los fondos que tiene destinados la UE para el desarrollo de zonas rezagadas. Adicionalmente, el ingreso al bloque europeo significaría un impulso aún mayor en la confianza de los inversionistas; así, es posible considerar un aumento en la IED. Pero, sin duda de que el gran objetivo es simple y trascendental: el acceso al mercado común que permitiría un aumento significativo del volumen total del comercio, en gran medida con la desaparición de barreras arancelarias y no arancelarias. La competencia permitiría que los consumidores turcos tengan acceso a más bienes y servicios. Esto sin contar las cuantiosas reformas institucionales que en alguna evidencia obtenida por Lejour y De Mooij (2005) estiman que impactarían notablemente en el PIB nacional. En otras palabras, la sostenibilidad del éxito económico turco puede verse determinada por su capacidad de adherirse armónicamente a la UE.

Pero, los beneficios de la UE no pueden dejarse de lado y caminan por el lado de la geopolítica y la economía: Turquía es el vaso comunicante con el oriente, fuente de energía necesaria para el aparato productivo europeo y de unos mercados emergentes atractivos para una UE que requiere potenciar su capacidad exportadora. Hoy, Turquía debe demostrarle al bloque que los beneficios de su adhesión superan los costos, en gran medida determinados por la disparidad entre las dos partes en temas de ingreso y desarrollo. Uno de los primeros costos de la adhesión turca serían los que deben asumir el Fondo de Cohesión y el Fondo Europeo de Desarrollo Regional, que se sostienen por contribuciones de los países miembros. Por mandato, estos recursos deben destinarse a tres objetivos fundamentales: el desarrollo y el ajuste estructural que tiene una economía en comparación con la media europea; conversión económica de sectores con rezagos estructurales y; la modernización de los sistemas políticos, educativos, de seguridad social y para la generación de empleo. Por ahora, la pericia de la diplomacia turca está en demostrar que abrirse al oriente es una clave para el éxito de la UE.

\section{Conclusiones}

En la última década Turquía se ha transformado en gran medida por la estabilidad política mostrada -que contrasta con el entorno inestable de la región-, y la puesta en marcha de reformas estructurales, especialmente en su política fiscal, y generación de incentivos y políticas para la atracción de IED que, de acuerdo a la evidencia obtenida, 
muestra una relación positiva y de doble vía con el crecimiento del PIB. Finalmente, la orientación de la política internacional de este país hacia una eventual adhesión a la Unión Europea juega un papel fundamental para entender el panorama turco. En este sentido, la sostenibilidad de su éxito económico estará determinada por la capacidad de profundizar las reformas y de disuadir a los europeos de la conveniencia mutua de su adhesión. En gran medida, Turquía como mercado emergente requiere tanto de Europa como Europa necesita a Turquía como país miembro. Y esto no es en vano, las perspectivas de Turquía en los próximos 35 años señalan que su PIB será incluso superior al de los principales países de la UE.

\section{Bibliografía}

A. LEJOUR y R. A. De Mooij. Turkish delight: Does turkey's accession to the eu bring economic benefits? Kyklos, 91(58), 2005.

F. KAYAy S. Yilar. Fiscal transformation in turkey over the last two decades. OECD Journal on Budgeting, 2011. K.-J. Koch, E. Ilgun, y M. Orhan. How do foreign direct investment and growth interact in turkey? Eurasian Journal of Business and Economics, 3(6):41-55, 2010.

I. OTZURK y H. Kalyoncu. Foreign investment and growth: an empirical investigation based on cross-country comparison. Economia internazionale, 60:75-82, 2007.

M. BABAKR. Turkey's economic development: Luck or wise administration? Strategic Outlook, 2013. 